\title{
Magnetism of the He-weak star HR 2949†
}

\author{
Thomas Rivinius ${ }^{1}$, Gregg A. Wade ${ }^{2}$, Richard H. D. Townsend ${ }^{3}$, \\ Matthew Shultz ${ }^{2}$, Jason H. Grunhut ${ }^{2}$, Otmar Stahl ${ }^{4}$ \\ and the MiMeS collaboration \\ ${ }^{1}$ ESO, Chile; ${ }^{2} \mathrm{RMC}$, Canada; ${ }^{3} \mathrm{UW}$ Madison, USA; ${ }^{4} \mathrm{LSW} / \mathrm{ZAH}$ Heidelberg, Germany
}

\begin{abstract}
A magnetic field and rotational line profile variability $(l p v)$ is found in the He-weak star HR 2949. The field measured from metallic lines varies in a clearly non-sinusoidal way, and shows a phase lag relative to the morphologically similar He I equivalent width variations. The surface abundance patterns are strong and complex, and visible even in the hydrogen lines.
\end{abstract}

Keywords. stars: binaries, stars: early-type, stars: magnetic fields

\section{Introduction}

The mid-B star HR 2949 was for many years considered to be non-variable, and indeed was listed in standard star catalogues. However, Rivinius et al. (2003) noted that the star is actually variable both photometrically as well as spectroscopically. They classified it as a He-weak star. Here we report the discovery of a magnetic field and describe the line profile variations $(l p v)$ due to surface abundance inhomogeneities. The existence of these inhomogeneities was already proposed by Rivinius et al. (2003), but could not be investigated further on the basis of solely four spectra.

\section{Observations}

Eight archival spectra have been obtained with the FEROS instrument at La Silla, providing a resolving power of 48000 over a spectral range of 375 to $890 \mathrm{~nm}$. Spectropolarimetric (Stokes $V$ ) data were taken with ESPaDOnS at the $3.6 \mathrm{~m} \mathrm{CFHT} \mathrm{in} \mathrm{the}$ 2009/2010 season, in total 16 measurements. The observations were obtained as part of the Magnetism in Massive Stars (MiMeS) Large Program.

Koen \& Eyer (2002) quote a period of $P=1.9093 \mathrm{~d}$ from HIPPARCOS photometric data. Re-analysing these data using the Lomb-Scargle statistic, we obtain $P=1.9083 \pm$ $0.00025 \mathrm{~d}$. In the spectra, the helium lines show the strongest variability. The time base covered by the spectra is long, 1999 to 2009, but there is severe aliasing. However, the aliases are narrow and there is only one peak in reasonable agreement with the Hipparcos data, at $P=1.90871 \pm 0.00007 \mathrm{~d}$. As the epoch we chose the date of the ESPaDOnS measurement showing the most negative field, so that our adopted ephemeris is:

$$
T_{\operatorname{minB}}(\mathrm{HJD})=2455223.987+1.90871(7) \times E
$$

\section{Spectral Variations}

The magnetic field as measured by ESPaDOnS (using Least-Squares Deconvolution applied to metallic lines) confirms the photometric and spectroscopic periods. Figure 1 shows the respective phased variations. The field varies between -760 and $+200 \mathrm{G}$. There

$\dagger$ Based on observations under ESO programs 073.C-0337, 076.C-0164 and the MiMeS large program at the CFHT. 

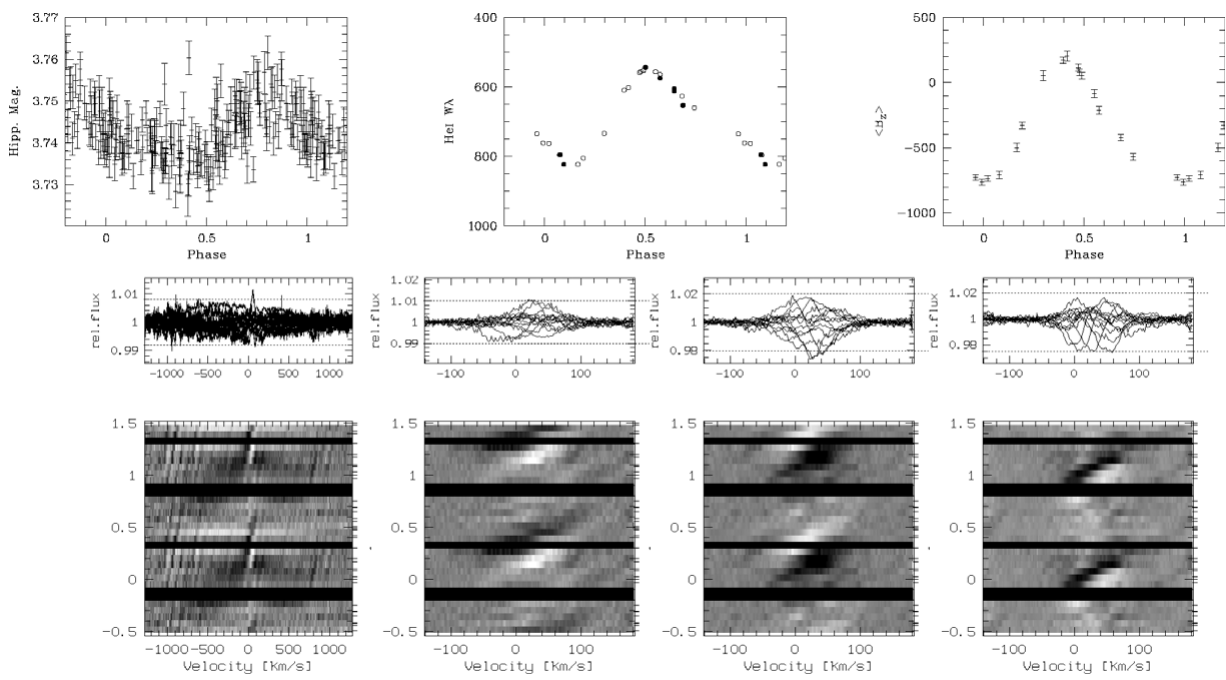

Figure 1. Upper row, from left to right: Hipparcos photometry, equivalent width variability of the combined He I 4009 \& 4026 lines (ESPaDOnS, open symbols; FEROS, filled symbols), and magnetic data. Lower row, left to right: Exemplary types of line profile variation seen in HR 2949: Balmer wings, Balmer cores (both H $\beta$ ), helium (He I 4388), metal (Fe I 4949).

is clear rotational line profile variability $(l p v)$, with no line in the spectrum unaffected. Taking the behavior of the He I lines as a starting point, the other lines can be sorted into three variability morphology groups: Lines similar to helium are those of $\mathrm{Mg}$ I 8807, Mg II 4481, O I 8446. They show a clear modulation of EW. Lines behaving inversely to He I, are Si II 4128 and Si III 4553, Cr II 4588, P II 6043, P III 4222, and the Balmer lines. Also these vary in EW with rotation. The majority of lines show a third pattern, e.g. S II 5640, Fe II 4949, C II 4267, Ca II 8662, Sr II 4215, Ti II 4564 and thus shall be called metal group behavior. While these lines show clear $l p v$, their EW is rather non-variable. Finally, some lines show different types of hybrid variation patterns.

It is noteworthy that the Balmer lines show a clear pattern. The line core shows an abundance-like pattern well described as an "anti-helium"-group behavior. We attribute the pattern to an actual variation of the $\mathrm{H}$-abundance, rather than to a change in local surface parameters. However, there are also changes in the Balmer line wings probably caused by local parameter variations. Although the effect is subtle, less than $0.5 \%$, and in spite of the notoriously difficult normalization of the Balmer wings in echelle data the pattern is identical in all lines.

\section{Conclusions}

HR 2949 is a magnetic star of the He-weak/Si-strong type. Its surface shows the clear signature of the typically complex abundance patterns characteristic to such stars. In addition to the abundance variation, the variability of the Balmer line wings indicates some other local parameter changes, such as pressure or temperature, linked to the magnetic field.

\section{References}

Koen, C. \& Eyer, L. 2002, MNRAS, 331, 45

Rivinius, T., Stahl, O., Baade, D., \& Kaufer, A. 2003, IBVS 5397 